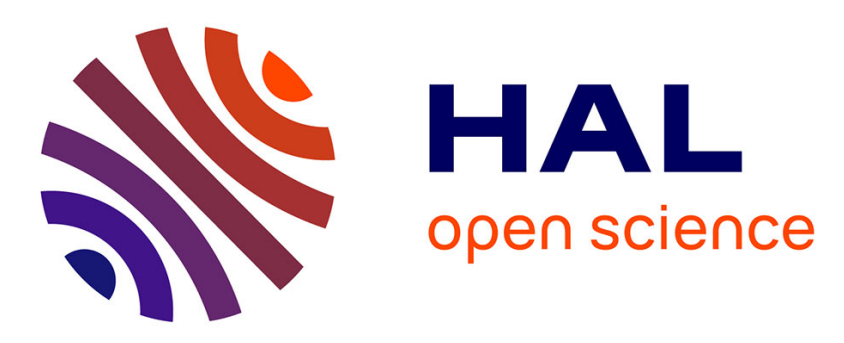

\title{
Fetal heart rate estimation from a single phonocardiogram signal using non-negative matrix factorization
}

Nafissa Dia, Julie Fontecave-Jallon, Pierre-Yves Guméry, Bertrand Rivet

\section{To cite this version:}

Nafissa Dia, Julie Fontecave-Jallon, Pierre-Yves Guméry, Bertrand Rivet. Fetal heart rate estimation from a single phonocardiogram signal using non-negative matrix factorization. EMBC 2019 - 41st Annual International Conference of the IEEE Engineering in Medicine and Biology Society, Jul 2019, Berlin, Germany. 10.1109/EMBC.2019.8857220 hal-02274618

\section{HAL Id: hal-02274618 https://hal.science/hal-02274618}

Submitted on 30 Aug 2019

HAL is a multi-disciplinary open access archive for the deposit and dissemination of scientific research documents, whether they are published or not. The documents may come from teaching and research institutions in France or abroad, or from public or private research centers.
L'archive ouverte pluridisciplinaire HAL, est destinée au dépôt et à la diffusion de documents scientifiques de niveau recherche, publiés ou non, émanant des établissements d'enseignement et de recherche français ou étrangers, des laboratoires publics ou privés. 


\title{
Fetal heart rate estimation from a single phonocardiogram signal using non-negative matrix factorization
}

\author{
Nafissa Dia, Julie Fontecave-Jallon, Pierre-Yves Gumery, Bertrand Rivet
}

\begin{abstract}
Fetal heart rate (FHR) is an important characteristic in fetal well-being follow-up. It is classically estimated using the cardiotocogram (CTG) non-invasive reference technique. However, this latter presents some significant drawbacks. An alternative non-invasive solution based on the fetal phonocardiogram (fetal PCG) can be used. But most of proposed methods based on the PCG signal need to detect and to label the fetal cardiac $S_{1}$ and $S_{2}$ sounds, which may be a difficult task in certain conditions. Therefore, in this paper, we propose a new methodology for FHR estimation from fetal PCG with one single cardio-microphone and without the distinction constraint of heart sounds. The method is based on the non-negative matrix factorization (NMF) applied on the spectrogram of fetal PCG considered as a source-filter model. The proposed method provides satisfactory results on a preliminary dataset of abdominal PCG signals. When compared to the reference CTG, correlation on FHR estimations between PCG and CTG is around $90 \%$.
\end{abstract}

\section{INTRODUCTION}

The monitoring of fetal well-being during labour and deliverance is based on the analysis of the fetal heart rate (FHR) and its variability, which allows to detect fetal distresses. The non-invasive reference technique used nowadays is the cardiotocogram (CTG) developed since the $60 s$ and based on ultrasounds. However, even if its use is the most common and represents $99 \%$ of cases in 2002 [1], CTG presents some limitations whose most important are confusion between maternal and fetal rhythms, and signal loss [2], [3].

There are non-invasive alternative solutions based on physiological cardiac signals [4] as the electrocardiogram (ECG) for cardiac electrical activity or the phonocardiogram (PCG) which gives access to mechanical heart information. The sensors are usually placed on the mother abdomen and signal processing is required to reach the fetal signals. Using ECG, heart rate is estimated classically for infants or adults by detecting $R$ peaks, which are the most prominent waves in ECG signals [5]. But, on abdominal ECG, the fetal signal is of very low power compared to the maternal one making difficult the $R$ peaks detection. Commonly, source separation is used to overcome this difficulty, based on independent component analysis [6], [7], [8]. However, this technique requires a lot of sensors placed on the mother thorax and

This work is supported by the French National Research Agency, as part of the SurFAO project (ANR-17-CE19-0012).

N Dia, J Fontecave-Jallon and P-Y Gumery are with Univ. Grenoble Alpes, CNRS, CHU Grenoble Alpes, Grenoble INP*, TIMCIMAG, Grenoble, France (phone: +33456520059; fax: +33456520033; email: nafissa.dia@univ-grenoble-alpes.fr). B Rivet is with Univ. Grenoble Alpes, CNRS, Grenoble INP*, GIPSA-lab, Grenoble, France (e-mail: bertrand.rivet@gipsa-lab.grenoble-inp.fr). * Institute of Engineering Univ. Grenoble Alpes

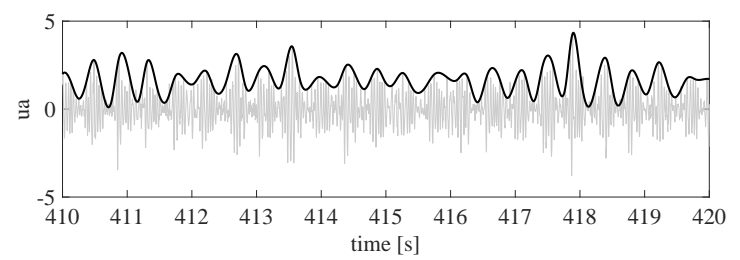

Fig. 1. Fetal PCG in gray line (abdominal PCG after simple 20-200 $\mathrm{Hz}$ band-pass filtering) and its envelop in black line

abdomen, non-compatible for a real clinical ergonomic setting, for the mother as well as for the medical staff.

Unlike abdominal ECG, fetal PCG is the most significant part recorded by abdominal microphones on pregnant women. Therefore, it is used increasingly to estimate the fetal cardiac frequency [9], [10]. Numerous methods of fetal PCG processing are presented in the state of the art and classified in different categories [4]. Among them, classical or conventional filtering methods allow to extract fetal phonocardiogram signal from abdominal recordings [11] but are often insufficient. Adaptive filtering technique [12] is also proposed but it suffers from a lack of robustness. In lineardecomposition group, one can cite methods based on timefrequency representation like short-time Fourier transform (STFT) [13], wavelets transform [14], which are efficient in signal analysis, and blind source separation (BSS) [15]. In addition, most of the existent PCG-based methods, as in [16], [17], need to locate and differentiate on the PCG signal envelop, the instants of $S_{1}$ and $S_{2}$ sounds, which are the most audible cardiac sounds and correspond to the closure of respectively the atrial-ventricular, and the aortic and pulmonary valves. But, as illustrated in Fig. 1 with one example of fetal PCG and its associated envelop, detecting and identifying $S_{1}$ and $S_{2}$ events may not be as easy in fetal PCG as in adults clean PCG, especially as signals are interfered by noises (such as gastric or liquid) and may be then roughly exploitable.

Based upon these limitations, we propose a novel methodology for FHR monitoring using one unique PCG sensor placed on the mother abdomen for an optimal ergonomic aspect, and which does not require the specific annotation of $S_{1}$ and $S_{2}$ patterns during processing. The proposed methodology is based on the non-negative matrix factorization (NMF) [18], [19], [20] algorithm applied on the spectrogram of the fetal PCG. First the methodology will be described, then the obtained FHR estimation will be evaluated on real fetal PCG data and partially compared to CTG reference. 


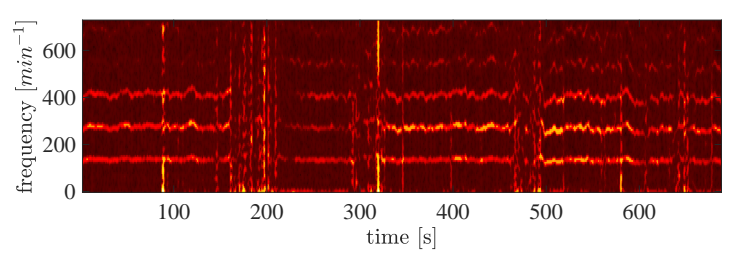

Fig. 2. Spectrogram of a fetal PCG $\left(F_{s}=1 \mathrm{kHz}\right.$, window $=4 s$, shift $=$ $32 \mathrm{~ms}$, zero-padding ratio $=4$ )

\section{MATERIALS AND FETAL HR ESTIMATION}

In this section, the signals database used for the evaluations is described (Section II-A) before introducing the modeling of the PCG signal (Section II-B) and the proposed method to estimate the FHR from PCG (Section II-C).

\section{A. Signals acquisition and description}

Four volunteer women participated in the study. They presented a pregnancy with normal progression between 38 and 39 weeks of gestation. They provided informed consent and the study was approved by the relevant ethics committee (CHU Grenoble Alpes). Participants were in supine and comfortable position. For each woman, the position of the fetus was checked, especially the side of the fetal back, by an obstetrical ultrasound scan. This allowed the PCG sensor to be placed in an optimal position. The woman was then equipped with one cardio-microphone (MLT201, ADInstruments) placed and kept by a belt on the abdomen in front of the fetal heart for abdominal PCG acquisition. Abdominal PCG signals were sampled at $1 \mathrm{kHz}$ and preprocessed by a $20-200 \mathrm{~Hz}$ band-pass filter. In parallel, a CTG reference for FHR was simultaneously recorded using the Oxford system CTG (Sonicaid Team Duo).

\section{B. Source-filter model of the fetal PCG}

Our proposed algorithm is based on NMF applied on the spectrogram of abdominal PCG (Fig. 2). As expected, by computing the spectrogram from the short-time Fourier transform (STFT) using a long enough window (so that several heart beats are considered), the spectrogram highlights an harmonic structure due to quasi-periodic aspect of physiological signals (Fig. 1): note the harmonics occurring of the fundamental frequency and the time-varying value of this later. Indeed, it is classical to model a quasi-periodic signal as a time-varying source-filter model. For each time window of the STFT, the excitation part is a Dirac comb at the rhythm of heart beats repetition (i.e. at the cardiac frequency), convolved by a filter part, representing the shape of the heart beats. Both terms evolve with time since both the cardiac frequency is not constant and the shape of each heart beat can be different.

Let $X(f, t)$ be the modulus of the STFT of the abdominal PCG at time $t$ and frequency $f$. It can be expressed, following the (time-varying) source-filter model, as

$$
\forall(f, t), \quad X(f, t)=X^{(e)}(f, t) X^{(\varphi)}(f, t),
$$

where $X^{(e)}(f, t)$ (resp. $\left.X^{(\varphi)}(f, t)\right)$ is the modulus of the STFT of the excitation (resp. the filter), which can be recast as

$$
\mathbf{X}=\mathbf{X}^{(e)} \odot \mathbf{X}^{(\varphi)},
$$

$\mathbf{X} \in \mathbb{R}_{+}^{F \times N}, \mathbf{X}^{(e)} \in \mathbb{R}_{+}^{F \times N}$ and $\mathbf{X}^{(\varphi)} \in \mathbb{R}_{+}^{F \times N}$, with $F$ the number of frequency bins and $N$ the number of time windows. $\odot$ is the Hadamard product (i.e. the element-wise multiplication) and $\mathbb{R}_{+}$is the non-negative real numbers set.

\section{FHR estimation based on NMF}

In view of the spectrogram structure, it is common to use the NMF for its analysis and interpretation. Indeed, the NMF is a matrix decomposition algorithm that allows to factorize the spectrogram as a product of matrices of lower rank [18], [19]. Applying the NMF on the spectrogram of our sourcefilter model leads to [20], [21]

$$
\mathbf{X} \simeq\left(\mathbf{W}^{(e)} \mathbf{H}^{(e)}\right) \odot\left(\mathbf{W}^{(\varphi)} \mathbf{H}^{(\varphi)}\right),
$$

where $\mathbf{W}^{(e)} \in \mathbb{R}_{+}^{F \times K_{e}}$ (resp. $\mathbf{W}^{(\varphi)} \in \mathbb{R}_{+}^{F \times K_{\varphi}}$ ) is the spectral matrix of the excitation (resp. the filter) and $\mathbf{H}^{(e)} \in \mathbb{R}_{+}^{K_{e} \times N}$ and $\mathbf{H}^{(\varphi)} \in \mathbb{R}_{+}^{K_{\varphi} \times N}$ are their related temporal amplitudes $\left(K_{e}<\min (F, N)\right.$ and $\left.K_{\varphi}<\min (F, N)\right)$. To take into account the harmonic structure, each column of excitation dictionary $\mathbf{W}^{(e)}$ is a Dirac comb whose fundamental frequency is chosen in a range of possible fetal HRs, typically between 30 and 300 beats per minutes (bmp). Therefore $\mathbf{H}^{(e)}$ has to select, at each instant $t$, the more consistent cardiac frequency among all found in the dictionary. $\mathbf{W}^{(\varphi)}$ allows to estimate the spectral envelop of the fetal PCG and the matrix $\mathbf{H}^{(\varphi)}$ contains its temporal evolution. These matrices, except $\mathbf{W}^{(e)}$ which is fixed, are iteratively updated thanks to our NMFbased algorithm whose details are given in [21].

The FHR is carried by $\mathbf{H}^{(e)}$ and is estimated by extracting the cardiac frequency corresponding to the maximum amplitude in each column of $\mathbf{H}^{(e)}$ :

$$
F H R(t)=v\left(\arg \max _{k} H_{k, t}^{(e)}\right)
$$

where $H_{k, t}^{(e)}$ is the $(k, t)$-th entry of $\mathbf{H}^{(e)}$ and $v \in \mathbb{R}^{K_{e}}$ is the vector of the $K_{e}$ fundamental frequencies of each Dirac comb of $\mathbf{W}^{(e)}$. Nevertheless, FHR values estimated by (4) do not all correspond to the cardiac frequency at each time but can correspond to one of its harmonics or sub harmonics as shown in the results section (Section III-A). To propose a more robust estimation of the FHR, a post-processing is applied on the FHR (4) to detect if it is a (sub-)harmonic of the cardiac frequency and then to correct it. The detection of the (sub-)harmonic FHR estimation from $F H R(t)$ is based on its derivative and the comparison with a average FHR as detailed below. As one can see on Fig. 3, when the FHR is detected as its second harmonic or its half sub-harmonic, the relative derivative of $F H R(t)$ defined as

$$
\Delta F H R(t)=\frac{F H R(t)-F H R(t-1)}{F H R(t-1)},
$$

presents some jumps equal to 1 and -0.5 which mark the beginning and the end of each wrong estimation. However, 

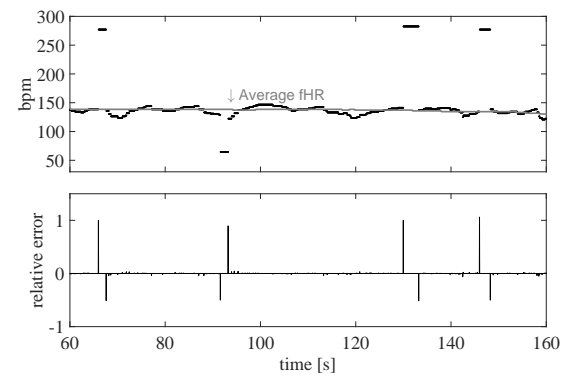

Fig. 3. Algorithm of post-processing. Top: FHR estimation (4) (black points) and the average FHR $F H R_{r e f}(t)$ (grey line). Bottom: FHR relative derivative (5).

both values can be related to a begin or to an end. To disambiguate them, a reference average $\operatorname{FHR} F H R_{r e f}(t)$ is computed as the moving median of $F H R(t)$ on the last minute, and is compared to $F H R(t)$ to provide a corrected FHR estimation $F H R^{*}(t)$ by the following scheme:

- if $\Delta F H R(t) \in[.8,1.2]$ and $0.8 F H R_{r e f}(t) \leq F H R(t)$ then this jump is classified as the begin of the detection of the second harmonic of the cardiac frequency. This segment ends when $\Delta F H R(t) \in[-.6,-.4]$ and $-0.1 \leq$ $\frac{F H R(t)-F H R_{\text {ref }}(t)}{F H R_{\text {ref }}(t)} \leq 0.1$. For all the values inside this segment, $F H R^{*}(t)=F H R(t) / 2$.

- else if $\Delta F H R(t) \in[-.6,-.4]$ and $F H R(t) \leq$ $F H R_{\text {ref }}(t) / 2$, then this jump is classified as the begin of the detection of the half sub-harmonic of the cardiac frequency. This segment ends when $\Delta F H R(t) \in[.8,1.2]$ and $-0.1 \leq \frac{F H R(t)-F H R_{r e f}(t)}{F H R_{r e f}(t)} \leq 0.1$. For all the values inside this segment, $F H R^{*}(t)=2 F H R(t)$.

- else there is no error and $F H R^{*}(t)=F H R(t)$.

\section{RESULTS AND DISCUSSIONS}

The following paragraphs firstly present the results obtained by our proposed NMF-based algorithm for FHR estimation and then discussions and perspectives that follow.

\section{A. Accuracy of $\mathbf{H}^{(e)}$ and fetal heart rate estimation}

Figure 4 shows an example of FHR estimated by our proposed algorithm. The estimation of $\mathbf{H}^{(e)}$ highlights a timevarying fundamental frequency around $140 \mathrm{bpm}$, corresponding to a classical average value for FHR. The FHR estimation obtained according to (4) is depicted in the second row of Fig. 4: one can observe some outliers around $300 \mathrm{bpm}$ due to selection of the second harmonic instead of fundamental component. FHR estimation after post-processing is presented in the third row of Fig. 4, where we can notice that some cardiac frequencies have been rectified, reducing therefore the number of outliers.

Figure 5 illustrates the behaviour of the proposed approach to estimate the FHR. Three parts are highlighted (white rectangular windows) to show three typical situations. Window a) shows a fetal PCG where $S_{1}$ and $S_{2}$ sounds are hardly identifiable. Window b) highlights fetal PCG where $S_{1}$ and $S_{2}$ sounds are recognizable and window c) illustrates
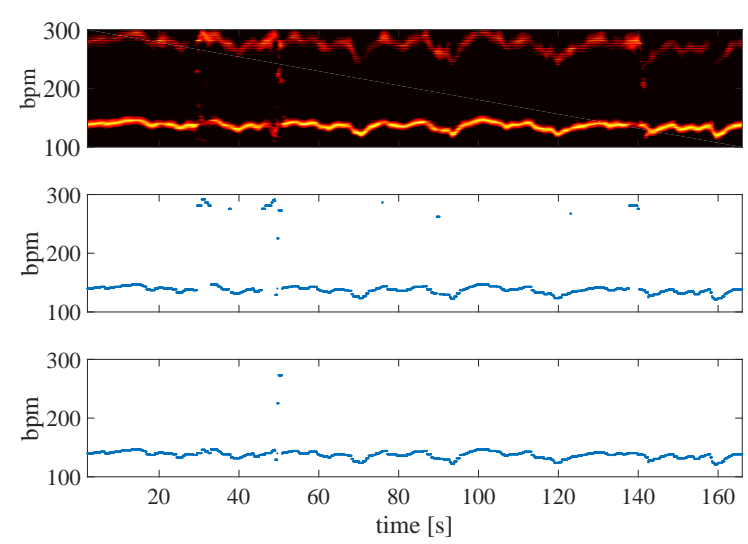

Fig. 4. Estimation of FHR for volunteer $V_{4}$. From top to bottom: matrix $\mathbf{H}^{(e)}$ estimated according to our proposed NMF-based algorithm, FHR estimation (4) extracted from $\mathbf{H}^{(e)}$ and FHR after post-processing step.

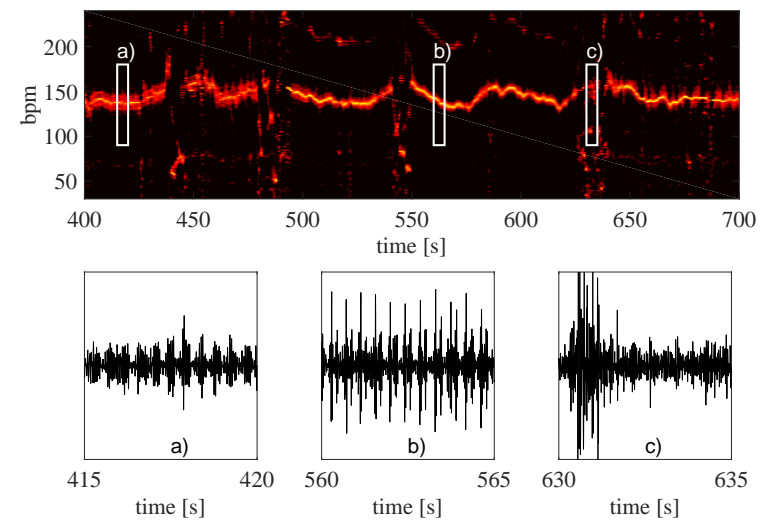

Fig. 5. $\mathbf{H}^{(e)}$ estimated according to our proposed NMF-based algorithm for volunteer $V_{1}$ (first row). Illustration of various behaviors of the corresponding temporal fetal PCG signal at different instants (second row) : a) with $S_{1}$ and $S_{2}$ sounds hardly identifiable, b) with $S_{1}$ and $S_{2}$ sounds recognizable and c) with important noise

a very noisy fetal PCG. Our proposed algorithm is obviously efficient when $S_{1}$ and $S_{2}$ sounds are easily recognizable (Fig. 5b) but also when both constitute only one pattern with noise (Fig. 5a). This is valuable compared to some classical methodologies based on PCG envelop detection which need to locate $S_{1}$ and $S_{2}$ sounds. However, when the signal is really noisy (Fig. 5c), our proposition gets still some difficulties for an accurate estimation of FHR.

\section{B. FHR estimation quantitative evaluations}

To quantify the accuracy of the FHR estimation by our proposed method, the post-processed FHR is compared to the one provided by the synchronous CTG (Fig. 6) for two volunteers $\left(V_{1}\right.$ and $\left.V_{2}\right)$. In both cases, one can notice that the FHR estimation from CTG and from our proposed NMF-based algorithm are very similar and follow the same variations and variability. For these 2 volunteers, correlation between FHR from CTG and FHR from PCG is respectively evaluated at $91 \%$ for $V_{1}$ and $84 \%$ for $V_{2}$. Unfortunately, CTG was not exploitable for volunteer $V_{3}$, due to a problem of synchronization, and acquisition for volunteer $V_{4}$ has been 

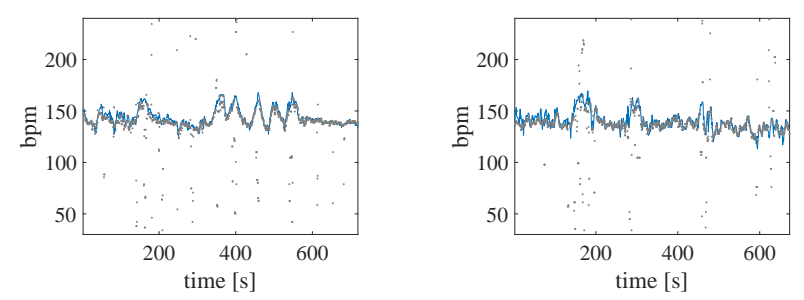

Fig. 6. Comparison between FHR estimation from fetal PCG (in black points) and FHR estimation from reference CTG (in blue line) for two volunteers $V_{1}$ (left) and $V_{2}$ (right)

made without CTG. In addition, complementary quantitative evaluation has been made for the four volunteers and is depicted on Table I. It reports the ratio of outliers defined as the FHR estimations that differ from the reference average FHR $F H R_{r e f}(t)$ by more than $10 \%$ divided by the total number of FHR estimations. This ratio is evaluated between $0.6 \%$ and $14.3 \%$ for the 4 volunteers, which highlights that FHR estimations are consistent in almost $90 \%$ of cases.

TABLE I

RECORDING DURATION AND OUTLIERS' RATIO FOR THE 4 VOLUNTEERS

\begin{tabular}{|c||c|c|c|c|}
\hline $\mathrm{N}^{\mathrm{o}}$ of volunteer & $V_{1}$ & $V_{2}$ & $V_{3}$ & $V_{4}$ \\
\hline Recording duration $[s]$ & 865 & 690 & 65 & 168 \\
\hline Ratio of outliers $[\%]$ & 9 & 9.6 & 14.3 & 0.6 \\
\hline
\end{tabular}

\section{Discussions and perspectives}

These preliminary results on a few volunteers are promising since our proposed approach shows up a good correlation between the FHR estimated from CTG and PCG while the PCG signal may be sometimes complicated. But this has to be confirmed on more volunteers. In that purpose, a complete campaign of evaluation is planned as new acquisitions are considered in the context of larger clinical protocols.

From a methodological point of view, even if the proposed algorithm seems to be of high interest for FHR estimation, there are still some noisy segments of PCG signals where it fails. Some post-processing steps allow to correct some wrong estimations, but there is still tracks of improvements. Therefore, we propose on one hand to enhance the proposed methodology by taking into account detection issue related to the frequency dictionary (i.e. a better estimation of $\mathbf{H}^{(e)}$ ). On the other hand, it would also be interesting to combine synchronous abdominal PCG and ECG to take benefits of multimodality either by switching between modalities or by joining the NMF processing of both modalities, as information given by both can be complementary and/or redundant.

\section{CONCLUSION}

In this paper, we have proposed a novel approach for FHR estimation based on a source-filter model estimated by a NMF applied on the spectrogram. From a unique abdominal microphone and with a simple band-pass filtering pre-processing step, we are able to estimate a FHR with a certain robustness to noise. Moreover, comparisons with CTG reference recordings show that our estimations are coherent and correspond to the fetal cardiac rhythm. This promising simple procedure to estimate the FHR from a single abdominal microphone can be improved to achieve an even more accurate estimation than the proposed one by improving the estimation of $\mathbf{H}^{(e)}$ and the extraction of the FHR from it.

\section{REFERENCES}

[1] Anaes, "Intérêt et indications des modes de surveillance du rythme cardiaque ftal au cours de laccouchement normal," Gynecol Obstet Fertil, vol. 31, pp. 393-396, 2003.

[2] V. Equy, S. Buisson, M. Heinen, J.-P. Schaal, P. Hoffmann, and F. Sergent, "Confusion between maternal and fetal heart rate during the second stage of labour," Br J Midwifery, vol. 20(11), no. 11, pp. 794-798, 2012.

[3] K. Bhogal and J. Reinhard, "Maternal and fetal heart rate confusion during labour," Br J Midwifery, vol. 18, no. 7, pp. 424-428, 2010.

[4] P. C. Adithya, R. Sankar, W. A. Moreno, and S. Hart, "Trends in fetal monitoring through phonocardiography: Challenges and future directions," Biomed Sig. Proc. Control, vol. 33, pp. 289-305, 2017.

[5] J. Pan and W. J. Tompkins, "A real-time qrs detection algorithm," IEEE Trans Biomed Eng, vol. 32, no. 3, pp. 230-236, 1985.

[6] L. De Lathauwer, B. De Moor, and J. Vandewalle, "Fetal electrocardiogram extraction by blind source subspace separation," IEEE Trans Biomed Eng, vol. 47, no. 5, pp. 567-572, 2000.

[7] V. Zarzoso and A. K. Nandi, "Noninvasive fetal electrocardiogram extraction: blind separation versus adaptive noise cancellation," IEEE Trans Biomed Eng, vol. 48, no. 1, pp. 12-18, 2001.

[8] R. Sameni, "Extraction of fetal cardiac signals from an array of maternal abdominal recordings," Ph.D. dissertation, Institut National Polytechnique de Grenoble-INPG; Sharif University of , 2008.

[9] A. Jiménez, M. Ortiz, M. Peña, S. Charleston, R. González, A. Aljama, and S. Carrasco, "Performance of a method to generate fetal cardiotachograms using fetal phonocardiography," Comput Cardiol, pp. 453 456, 2001.

[10] F. Kovács, M. Torok, and I. Habermajer, "A rule-based phonocardiographic method for long-term fetal heart rate monitoring," IEEE Trans Biomed Eng, vol. 47, no. 1, pp. 124-130, 2000.

[11] H. E. Bassil and J. H. Dripps, "Real time processing and analysis of fetal phonocardiographic signals," Physiol Meas, vol. 10, no. 4B, p. 67,1989 .

[12] R. A. Pretlow III and J. W. Stoughton, "Signal processing methodologies for an acoustic fetal heart rate monitor," M.S. thesis, Old Dominion Univ., Norfolk, VA, US, 1991.

[13] A. Mittra and N. Choudhari, "Time-frequency analysis of foetal heart sound signal for the prediction of prenatal anomalies," J Med Eng Technol, vol. 33, no. 4, pp. 296-302, 2009.

[14] S. R. Messer, J. Agzarian, and D. Abbott, "Optimal wavelet denoising for phonocardiograms," Microelectronics J, vol. 32, no. 12, pp. 931941, 2001.

[15] A. Jimenez-Gonzalez and C. James, "Blind source separation to extract foetal heart sounds from noisy abdominal phonograms: A single channel method," in IET MEDSIP.

[16] M. Ruffo, M. Cesarelli, M. Romano, P. Bifulco, and A. Fratini, "An algorithm for fhr estimation from foetal phonocardiographic signals," Biomed Signal Process Control, vol. 5, no. 2, pp. 131-141, 2010.

[17] F. Kovcs, C. Horvth, dm T. Balogh, and G. Hossz, "Fetal phonocardiographypast and future possibilities," Comput Methods Programs Biomed, vol. 104, no. 1, pp. $19-25,2011$.

[18] P. Paatero and U. Tapper, "Positive matrix factorization: A nonnegative factor model with optimal utilization of error estimates of data values," Environmetrics, vol. 5, no. 2, pp. 111-126, 1994.

[19] D. D. Lee and H. S. Seung, "Learning the parts of objects by nonnegative matrix factorization," Nature, vol. 401, no. 6755, p. 788, 1999.

[20] J. Durrieu, A. Ozerov, C. Févotte, G. Richard, and B. David, "Main instrument separation from stereophonic audio signals using a source/filter model," in IEEE EUSIPCO, Aug 2009, pp. 15-19.

[21] N. Dia, J. Fontecave, P.-Y. Gumery, and B. Rivet, "Heart rate estimation from phonocardiogram signals using non-negative matrix factorization," Proc. IEEE ICASSP, 2019, (Accepted for publication). 